\title{
Influence of temperature on luminescent coupling and material quality evaluation in inverted lattice-matched and metamorphic multi-junction solar cells
}

\author{
M. Ochoa , M. A. Steiner , I. García ，J.F. Geisz , D. J. Friedman and C. Algora
}

\begin{abstract}
Inverted metamorphic multi-junction solar cells have reached efficiencies close to $46 \%$. These solar cells contain very highquality materials that exhibit strong luminescent coupling between the junctions. The presence of luminescent coupling has a significant impact on the behavior of multi-junction solar cells affecting the optimal design of these devices. Because of the importance of studying devices under real operating conditions, the temperature dependence of the luminescent coupling is analyzed over a range of $25-120^{\circ} \mathrm{C}$. Luminescent coupling analysis results show a reduction of the luminescent coupling current as a function of temperature in two tandem components of an inverted metamorphic triple junction solar cell such as $\mathrm{GaInP} / \mathrm{GaAs}$ and $\mathrm{GaAs} / \mathrm{GaInAs}$ solar cells. This reduction is quantified and examined by means of luminescent coupling analysis and modeling, electroluminescence measurements and optical modeling at the device and subcell level. The results of the models are verified and discussed.
\end{abstract}

KEYWORDS

III-V multijunction solar cells; temperature; luminescent coupling; material quality

\section{INTRODUCTION}

The operating temperature in a solar cell has a strong impact on its performance and reliability [1]. In a photovoltaic system, the cell temperature is affected by many factors such as ambient temperature, irradiance, wind speed, and the heat extraction properties of the module $[2,3]$. The accurate measurement of the cell temperature is a difficult task, but, in most cases, the estimated operating temperature of multi-junction solar cells working under concentrated illumination is in the range of $50-80^{\circ} \mathrm{C}$ [4]. In some cases, especially for large cells, the cell can reach temperatures above $100^{\circ} \mathrm{C}$ [5]. Therefore, the design of concentrator solar cells must consider its real operating temperature in order to maximize the electrical output in the field.

Different physical phenomena occur when the cell temperature increases, namely: (1) reduction of the bandgaps of the semiconductors in each subcell that determines how much light is absorbed in a subcell and how much light is transmitted to the underlying subcells thus changing the photocurrent of the multi-junction cell; (2) decrease of the open-circuit voltage as a result of the reduction in bandgap; (3) variation of the refractive index and absorption coefficient; (4) change in the majority free-carrier concentration affecting the electrical and thermal conductivity; (5) depletion of deep level defects that increases the non-radiative recombination current; (6) decrease in the minority carrier diffusion length, mobililites, and so on. In the end, all these variations result in a worse solar cell behavior at high temperatures.

Recently, inverted metamorphic (IMM) multi-junction solar cells have reached efficiencies close to $46 \%$ [6] and are candidates to surpass $50 \%$. The influence of temperature on these cells was studied by Steiner, et al. [7] who showed the efficiency of a triple-junction (3J) IMM solar cell at moderate concentration drops by around $6 \%$ absolute when the cell temperature increases from 25 to $126^{\circ} \mathrm{C}$. The efficiency of IMM and other III-V multijunction cells can be increased as a result of luminescent 
coupling (LC). The consideration of LC affects the structural optimization of the solar cell in terms of critical aspects such as thicknesses and bandgaps of the subcells [8,9]. None of the aforementioned papers tackled the effect of temperature on LC, for either the IMM or any other III-V multi-junction cells.

Accordingly, this paper contributes to fill this knowledge gap by analyzing the dependence of LC with temperature within a range typical for real operating conditions $\left(25-120^{\circ} \mathrm{C}\right)$. Two tandem components of an IMM $3 \mathrm{~J}$ solar cell have been analyzed: (1) $\mathrm{GaInP} / \mathrm{GaAs}$ (lattice-matched) and (2) GaAs/GaInAs (metamorphic) solar cells. We use the modeling and characterization techniques described in references $[10,11]$ to examine the $\mathrm{LC}$, complemented by electroluminescence (EL) measurements and optical modeling $[12,13]$ to examine the performance of the device in the presence of $\mathrm{LC}$ and to determine the quality of the subcells [14].

\section{EXPERIMENTAL}

Two inverted, dual junction structures, namely ML732 and MM708, were grown in an atmospheric pressure metalorganic vapour phase epitaxy (MOVPE) reactor [15]. ML732 is a $\mathrm{Ga}_{0.51} \mathrm{In}_{0.49} \mathrm{P} / \mathrm{GaAs}$ tandem grown lattice-matched on GaAs, and the MM708 is a $\mathrm{GaAs} / \mathrm{Ga}_{0.75} \operatorname{In}_{0.25}$ As metamorphic tandem grown on GaAs as shown in Figure 1. The solar cells were manufactured using conventional photolithography and metal deposition techniques, which details can be found in [15]. A $\mathrm{MgF}_{2} \mathrm{ZnS}$ ARC was deposited onto the ML732 device by thermal evaporation, while no ARC was deposited onto the MM708 device. The GaInP top cell in the ML732 device has the rear-heterojunction design described in [13], which has been demonstrated to lower the space charge region recombination. The other two subcells (GaAs and metamorphic GaInAs) follow traditional designs [15]. In order to enhance photon recycling and $\mathrm{LC}$ inside these solar cells, the substrate was removed in both structures, and a highly reflective gold mirror was electroplated at the back side of each cell. A highly doped $\mathrm{Al}_{0.3} \mathrm{Ga}_{0.7} \mathrm{As}$ layer for the ML732 and a $1 \mathrm{eV}$ GaInAs layer for the MM708 were grown to achieve a p-type ohmic contact with gold.

In addition to the LC analysis, the solar cells were characterized by QE and EL as described in $[11,13,16]$. The short-wavelength artifact in the QE measurement caused by LC was corrected by following the procedure shown in [11]. EL has been used to calculate the subcell dark J-V curves by using Rau's reciprocity theorem [17], and following the procedure in $[13,18]$ verify and confirm the LC results by using the subcell dark J-V curves and analyze the structures at the subcell level in the presence of LC [14,18].

All characterization data were taken at nominal temperatures of $25,50,75,100$, and $120^{\circ} \mathrm{C}$. A calibrated platinum-RTD surface probe was used to monitor the temperature on the stage surface. Therefore, every value shown in the upcoming graphs corresponds to the actual temperature on the stage. In order to control the cell temperature during the QE measurements, a Wavelength Electronics LFI-3751 (Wavelength Electronics, Inc., Bozeman,USA) controller was used. A gold-plated copper sample stage was mounted on a thermoelectric heater with a $10 \mathrm{k} \Omega$ thermistor coupled to the copper block.

In order to extract the LC current and its related parameters, the short circuit current of the device is measured as a function of the light intensity, as described in $[10,11]$. Spectral conditions are varied to force the LC current between the two junctions to dominate the limiting photocurrent. An adjustable light simulator comprising a Xenon lamp and high-brightness LEDs with wavelengths 470 , 740 and $850 \mathrm{~nm}$ was used to construct the simulated spectrum to selectively vary the light intensity in the GaInP and GaAs subcells, respectively. For every data point, the intensity on each junction relative to the ASTM G173 direct solar spectrum $\left(X_{i}\right.$ or equivalent suns for junction $i$, where $i=1$ corresponds to the top cell) was determined by measuring the photocurrent on a corresponding calibrated single junction reference cell, and correcting for any spectral mismatch using a spectral mismatch correction factor was calculated using the measured light

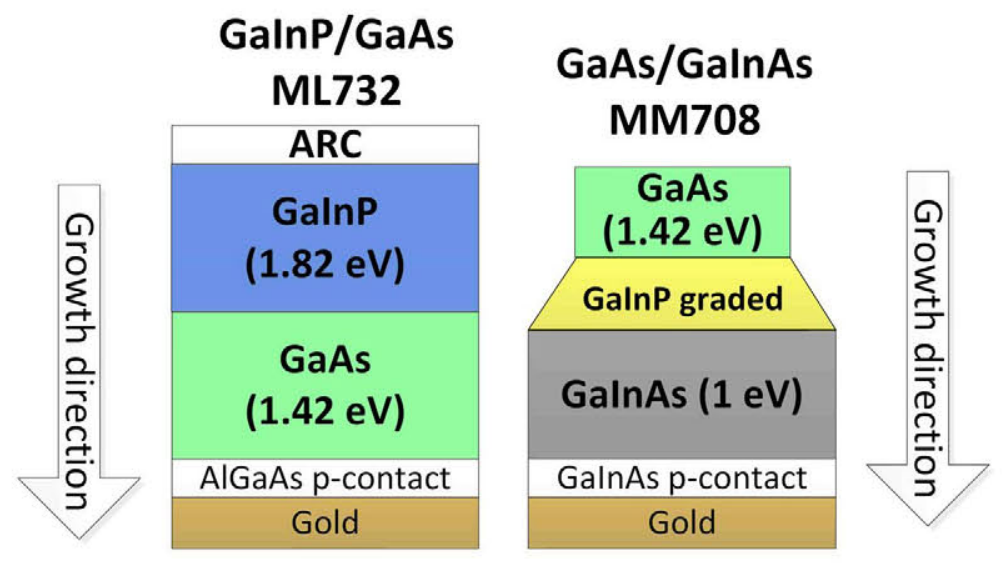

Figure 1. Design of the two structures analyzed. The active areas are 0.25 and $0.1 \mathrm{~cm}^{2}$ for ML732 and MM708, respectively. 
spectra and the quantum efficiency measurements [19]. The relative intensity of each junction was characterized as each LED was varied, and any spectral overlap between the LEDs was accounted for. The LED overlap was observed to be stronger as the temperature is increased, for the particular LED and cell bandgaps used, due to the bandgap temperature dependence. However, this overlap is well captured by the reference cell characterization (as shown in the next section). As mentioned in the preceding text, the LC current is measured by forcing the subcell under test to limit the tandem cell current in order to analyze the LC behavior. To force these conditions, the corresponding LED light source $\left(X_{1}\right.$ or $X_{2}$ for a dual junction solar cell case) is varied for the subcell under test from zero to the full range of the LED power, while the other subcells are under a fixed LED light power. The $J_{\mathrm{sc}}$ of the overall tandem cell is measured at every light bias step, and the $J_{\mathrm{sc}}$ versus equivalent suns curve is obtained. A detailed description of the measurement technique can be found in $[10,11]$.

Regarding the EL measurements, we followed the procedure described in $[13,18]$ using a high resolution spectroradiometer from Spectral Evolution (SR-3500). The EL spectrum is measured under a range of different forward current injections. EL also allows to deduce the external luminescent efficiency, which is an important metric to measure the quality of solar cells, and it is defined as the fraction of the total dark current recombination that leads to radiative recombination and emission of photons that escape out the front of the device [20]. The external radiative efficiency is calculated by integrating over the measured EL peak emission spectrum (in units of current density) of the subcell and dividing it by the injected current. More details on the measurement procedure can be found in $[14,18]$.

\section{RESULTS}

\subsection{QE results}

Figure 2a shows the external quantum efficiency (EQE) measurements for the $\mathrm{GaInP} / \mathrm{GaAs}$ two-junction cell (ML732) as a function of temperature. As expected, a shift of the cutoff wavelength in the EQE of each subcell is observed because of the temperature dependence of the bandgap in each material. The reflectance (Figure 2b) primarily shows differences with temperature near the bandgap of the bottom junction. This indicates that the refractive index does not show significant changes at any measured temperature. The plateau of the EQE response also shows very little changes (less than $1-2 \%$, which is within measurement uncertainty) indicating that the diffusion length is still greater than the cell thickness at high temperatures so as not to affect the carrier collection. Very similar behavior (Figure 3) was found for the GaAs/GaInAs two-junction cell (MM708).

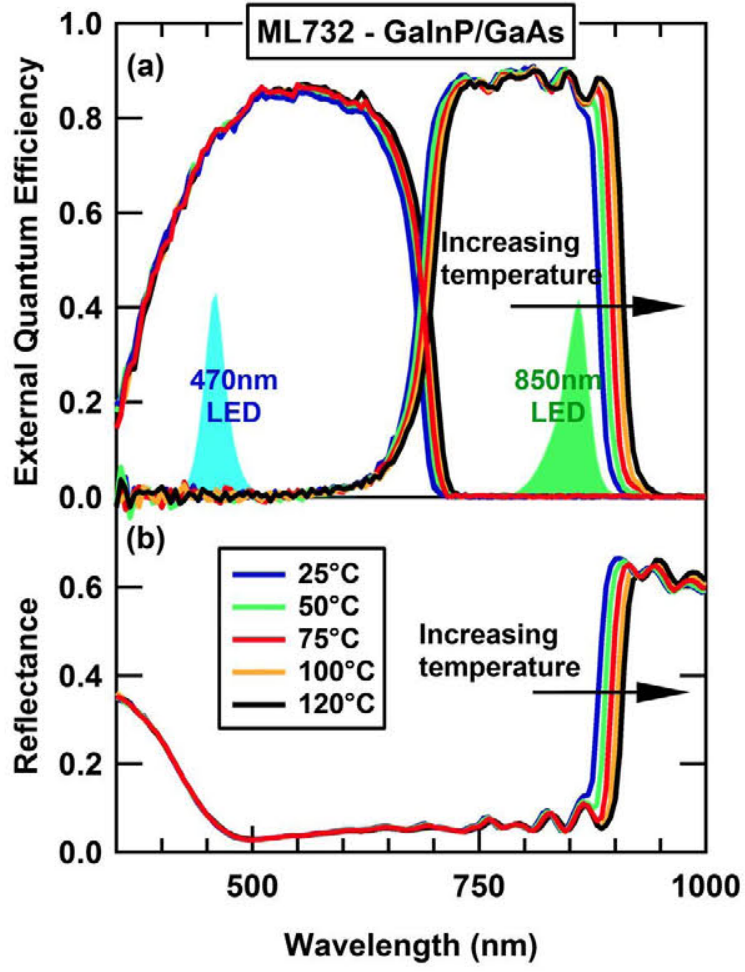

Figure 2. (a) External quantum efficiency measurements of the GalnP/GaAs two-junction cell (ML732) at different temperatures once corrected by luminescent coupling following the procedure described in [11]. (b) Reflectance measurements at different temperatures of the same solar cell.

\subsection{Luminescent coupling results}

Two different measurements are carried out in order to analyze the luminescent coupling: (1) with background LED light illuminating the bottom junction at around one-sun; and (2) without any background LED light on the bottom junction. The use of both measurements provides a more reliable extraction of the LC parameters as well as the photocurrents of the subcells relative to standard test conditions. Figures 4 and 5 show the measurements carried out to analyze the LC for GaInP/GaAs (ML732) and GaAs/GaInAs (MM708) cells as a function of temperature, for the cases with and without background light, and the fitting achieved by using the Steiner and Geisz model [10].

For GaInP/GaAs solar cell, the measurements with background light ( $X_{1}$ varied and $X_{2} \sim 0.9$ suns) show two different slopes as can be seen in the top graph of Figure 4. The first slope, on the left, corresponds to the top cell limiting the photocurrent until it reaches the background photocurrent generated by the bottom cell. At this point, the top cell is not limiting the current, so the limiting photocurrent corresponds to the bottom cell background photocurrent plus LC photocurrent. The bottom graph of Figure 4 shows clearly the LC where the bottom cell is not illuminated $\left(X_{1}\right.$ varied and $\left.X_{2}=0\right)$ and the LC dominates the photocurrent for the whole curve. As can be seen, 


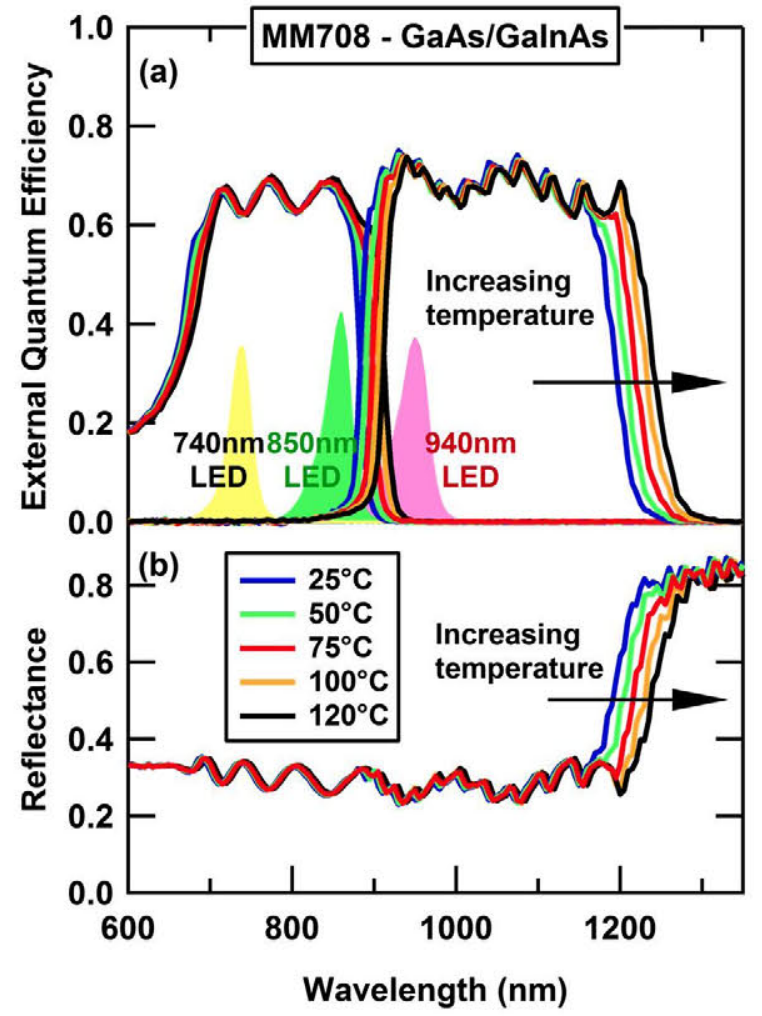

Figure 3. (a) External quantum efficiency measurements of the GaAs/GalnAs two-junction cell (MM708) at different temperatures once corrected by luminescent coupling following the procedure described in [11]. (b) Reflectance measurements at different temperatures of the same solar cell.

the slope of the lines is reduced as temperature increases, so the LC diminishes with temperature.

Figure 5 shows the radiative coupling from the GaAs top cell to the GaInAs bottom cell for the MM708 device. In the case with background LED light measurements (top graph in Figure 5, $\left.X_{2} \sim 0.75\right)$, LED overlap is present. The first slope ends at lower illumination intensity as temperature increases because of the increasing overlap of the $940 \mathrm{~nm}$ LED on the top cell. As temperature increases, the absorption range of each subcell extends to longer wavelengths, thus the GaAs cell absorbs more light from the $940 \mathrm{~nm}$ LED, and lower illumination is needed to make the bottom cell the limiting subcell. No major impact is expected from the LED overlap in the extraction of the LC properties because the LED intensities have been previously characterized by using appropriate reference cells. Finally, for $X_{2}=0$ (bottom graph of Figure 5), the curves that the LC dominate the $J_{\mathrm{sc}}$ and decreases with temperature.

\subsubsection{Luminescent coupling parameters as a function of temperature.}

Using a two-diode model with ideality factors of $n=1$ and $n=2$ in the absence of series resistance, shunting and reverse breakdown, the $\mathrm{LC}$ as a function of the relative
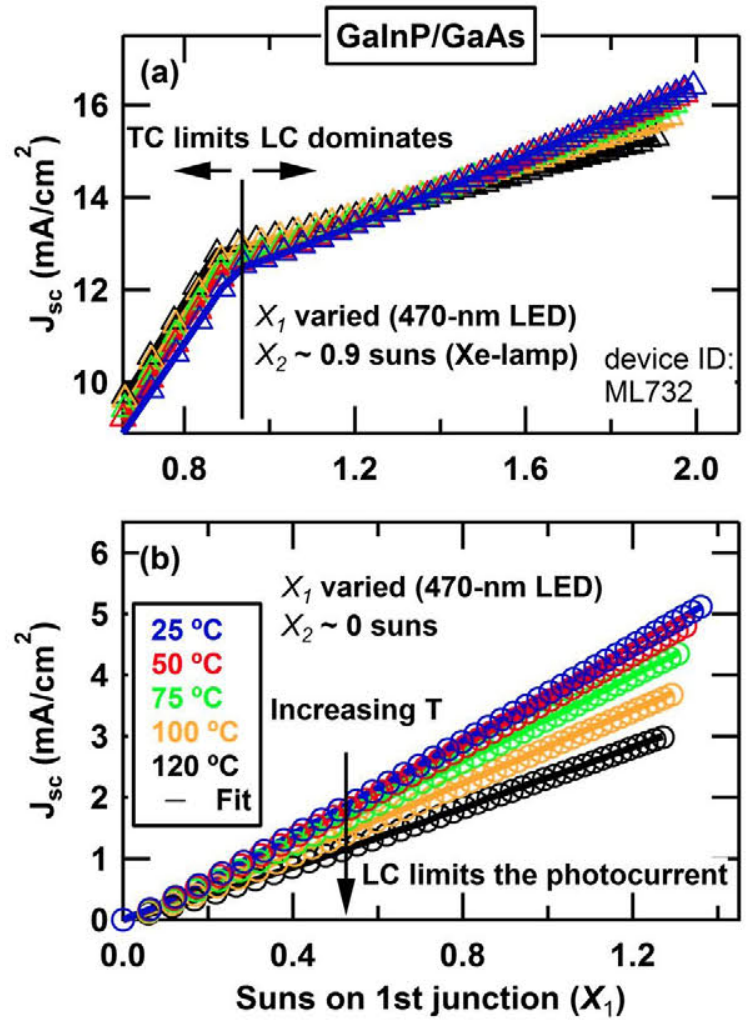

Figure 4. $J_{\mathrm{sc}}$ of GalnP/GaAs cell (ML732) at different temperatures for measurements (a) with background light $X_{2}$, and (b) without background light when $X_{1}$ is varied. The radiative coupling from the GalnP top cell is observed. Markers are data and lines are fits.

light intensity in each junction $\left(X_{i}\right)$ can be characterized by two experimentally measurable parameters [10]: the coupling efficiency, $\eta_{i, i+1}$ and $\varphi_{i}$. The first parameter evaluates the strength of the coupling by including optical effects (ability of photons to escape out of the subcell, absorption in layers between subcells, and so on) and the ratio between radiative and non-radiative components of the diffusion recombination current in the subcell emitting light. The second parameter $\left(\varphi_{i}\right)$ evaluates the linearity of the LC with light intensity from the upper junction and is given by $\varphi_{i}=\frac{J_{02}}{2 \sqrt{J_{01}}}$. Both parameters, $\eta_{i, i+1}$ and $\varphi_{i}$, determine the LC current, $J_{i, i+1}^{L C}$, in junction $i+1$ and is calculated by $[10]$

$$
J_{i, i+1}^{L C}=\eta_{i, i+1}\left[\sqrt{\varphi_{i}^{2}+J_{r e c}}-\varphi_{i}\right]^{2}
$$

where $J_{\text {rec }}$ is the recombination current density. Because $J_{\text {rec }}$ is a function of the total photocurrent density $\left(J_{i}^{\text {Total }}\right)$ in the device, its calculation depends on which cell is limiting the current. Because we are dealing with twojunction devices, the LC dominates when the second lower bandgap junction is limiting the photocurrent in the device. Therefore, $J_{r e c}=J_{e x t 1}-J_{e x t 2}-J_{12}^{L C}$ and Eq. (1) can be 

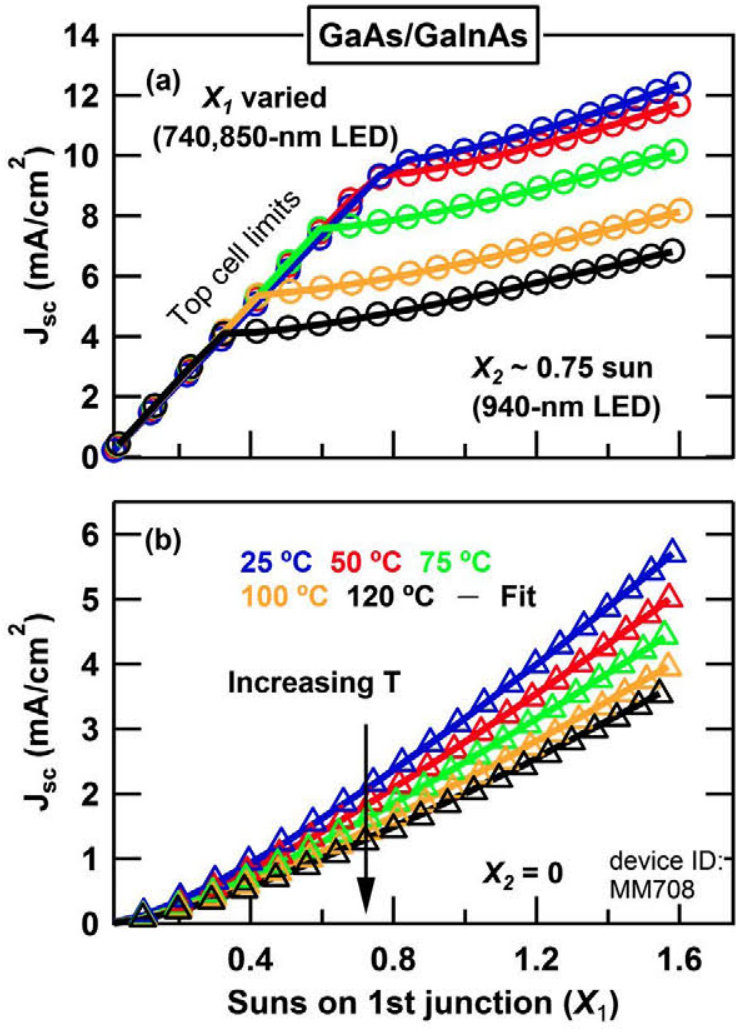

Figure 5. $J_{\mathrm{sc}}$ of GalnAs/GaAs solar cell (MM708) at different temperatures for measurements (a) with background light $X_{2}$, and (b) without the background light when $X_{1}$ is varied. The radiative coupling from GaAs to GalnAs is observed. Markers are data and lines are fits. Two LED light sources are used to ensure enough light for the measurements.

solved directly by using the externally induced photocurrents, $J_{e x t 1}$ and $J_{e x t 2}$ (previously characterized) on top and bottom junctions, respectively [10]:

$J_{12}^{L C}=\frac{\eta_{12}}{\left(1+\eta_{12}\right)^{2}}\left[\sqrt{\varphi_{i}^{2}+\left(1+\eta_{12}\right) \cdot\left(J_{e x t 1}-J_{e x t 2}\right)}-\varphi_{i}\right]^{2}$

In order to extract the LC parameters $\left(\eta_{i, i+1}\right.$ and $\left.\varphi_{i}\right)$ from the experimental data of Figures 4 and 5, the simplest case is setting $\varphi_{i}=0$, which means that the LC current is a function of black $\eta_{i, i+1} J_{\text {rec }}$ and can be explained by a single diode model $\left(J_{02}=0\right)$. However, even though there are some cases where $\varphi_{i}$ can be neglected (i.e., for a very high-quality cell and at a fixed temperature), we expect that the saturation current densities from the standard twodiode model, $J_{01}$ and $J_{02}$, change differently with temperature. Therefore, $\varphi_{i}$ will probably not remain constant with temperature and should be considered to be variable. On the other hand, the main uncertainty on the calculation of the LC parameters $\left(\varphi_{i}\right.$ and $\left.\eta_{i, i+1}\right)$ as a function of temperature is that multiple pairs of values of these parameters are able to obtain very good data fittings to the experimental measurements (the goodness of fit was evaluated by Chi-square tests where $\chi^{2}<0.01$ in each corresponding curve). Because of the existence of multi-solutions of the data fitting process for both $\varphi_{i}$ and $\eta_{i, i+1}$, it is compulsory to validate at least one of these LC parameters in order to provide more confidence to the model consistency. In this paper, we validate $\varphi_{i}$ by measuring the saturation current densities $\left(J_{01}\right.$ and $\left.J_{02}\right)$ which can be easily calculated or measured from EL. $\varphi_{i}(T)$ can be derived from the relationship between the saturation currents and temperature. Accordingly, by assuming proportionality in the relation of $D_{n, p} \tau_{n, p}^{-1}$ with temperature and using the temperature dependence of the intrinsic carrier concentration [21],

$$
n_{i}^{2} \propto T^{3} \cdot \exp \left(\frac{-E_{g}}{k T}\right)
$$

$J_{01}$ can be expressed as

$$
J_{01}(T) \cong \mathrm{q}\left(\frac{D_{n, p}}{\tau_{n, p}}\right)^{1 / 2} \frac{n_{i}^{2}}{N_{D, A}}=C_{1} \cdot T^{3} e^{-\frac{E_{g}}{k T}}
$$

where $C_{1}$ is a proportionality constant, $D_{n, p}$ the diffusion length of holes or electrons, $\tau_{n, p}$ the carrier lifetime of holes or electrons, $N_{D, A}$ the doping level of acceptors or donors and the other variables have their usual meaning. Assuming (i) the trap energy level is equal to the intrinsic Fermi level; (ii) the capture cross sections are equal for both carriers $\left(\sigma=\sigma_{n}=\sigma_{p}\right)$; and (iii) for $V>>k T / q$, the temperature dependence of $J_{02}$ arises from both $n_{i}$ and that of the lifetimes through the thermal velocity $\left(v_{t h}\right)$ [22]:

$$
J_{02}=\frac{\mathrm{qWn}_{i}}{2} \sigma v_{t h} N_{t}=C_{2} T^{2} \cdot \exp \left(\frac{-E_{g}}{2 k T}\right)
$$

where $C_{2}$ is a proportionality constant, $W$ is the depletion width, $N_{t}$ is the defects density and the other variables have their usual meaning. Finally, combining Eqs. (4) and (5) and solving for $\varphi_{i}$ yields

$$
\varphi_{i}^{2}(T) \propto \mathrm{T}
$$

Therefore, we proceed by using Eq. (6) to calculate the temperature dependence of $\varphi_{i}$ and then we use these $\varphi_{i}$ values as input data to calculate $\eta_{i, i+1}$. Accordingly, $\varphi_{i}$ and $\eta_{i, i+1}$ as a function of temperature are plotted in Figure 6 . The $\eta_{12}$ decreases by just $11 \%$ from 25 to $75^{\circ} \mathrm{C}$ and by $45 \%$ from 25 to $120^{\circ} \mathrm{C}$ in the case of the GaInP/GaAs cell (ML732). In the case of the GaAs/GaInAs cell (MM708), $\eta_{12}$ drops by $25 \%$ from 25 to $75^{\circ} \mathrm{C}$ and by $45 \%$ from the lowest to the highest temperature measured.

The possible values the LC parameters that can be well to fit the experimental data are within the colored regions around the dotted curves. In order to calculate this uncertainty (colored regions), we establish three different pairs of values that achieve reasonable good data fittings. 

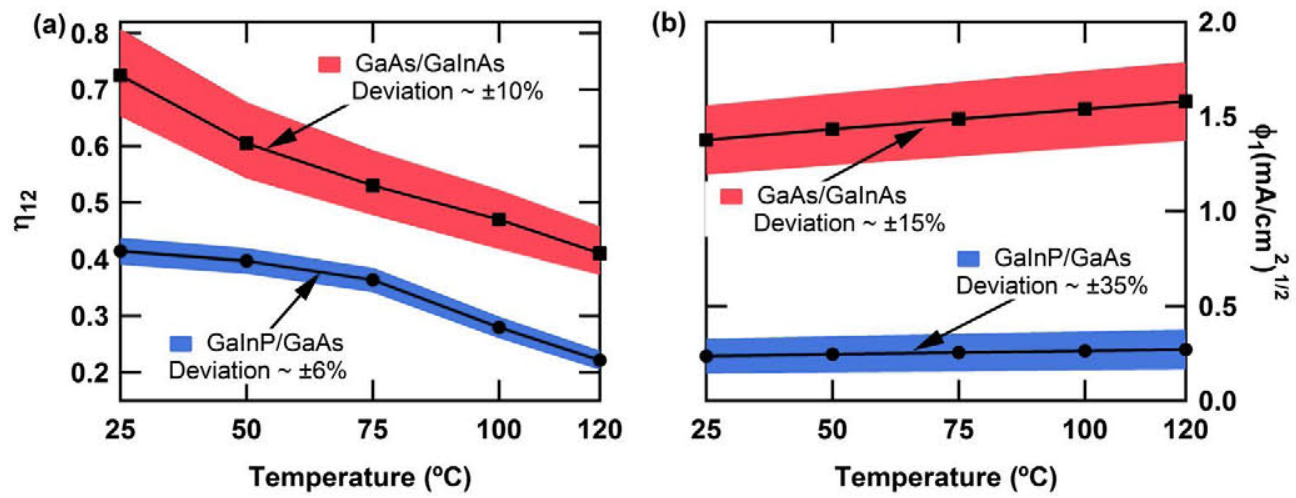

Figure 6. Luminescent coupling parameters: (a) $\eta_{12}$ and (b) $\varphi_{1}$ fitted from the measurements for the GalnP/GaAs cell, ML732 (blue region filled circles) and the GaAs/GalnAs cell, MM708 (red region and filled squares).

The black curves (1st pair of values) correspond to the best fitting, while the colored regions correspond to possible values that the LC parameters can take within lower (2nd) and upper (3rd) limits where the $\chi^{2}$ is closest to 0.01 . The black curve corresponding to $\eta_{12}$ has $\pm 6 \%$ of deviation for the $\mathrm{GaInP} / \mathrm{GaAs}$ cell and around $\pm 10 \%$ for the $\mathrm{GaAs} / \mathrm{GaInAs}$ cell, while $\varphi_{1}$ has a deviation of $\pm 35 \%$ and $\pm 15 \%$ for both cells, respectively. Figure 6 shows that a lower uncertainty in $\eta_{12}$ can be found for cases where $\varphi_{1}$ is relatively low. In cases where $\varphi_{1}$ is low (as in the case of GaInP in the ML732 device), its variation is high $( \pm 35 \%)$, and it has a lower deviation impact on $\eta_{12}( \pm 6 \%)$. This impact on $\eta_{12}$ increases $( \pm 10 \%)$ when $\varphi_{1}$ is higher even if its deviation is lower $( \pm 15 \%)$ as in the case of GaAs in the MM708 device. Therefore, a low $\varphi_{1}$ indicates a relatively small contribution from the space charge region (lower $J_{02}$ ), and $\eta_{12}$ becomes the dominant parameter. Despite the uncertainty, the trend of each parameter considering these deviations remains unchanged. For example, any value taken for $\varphi_{1}$ in the colored region of each structure in Figure $6 \mathrm{~b}$ yields similar trends for $\eta_{12}$ in Figure $6 \mathrm{a}$. Consequently, the use of $\varphi_{1}$ as an intermediate parameter in order to achieve robust values of $\eta_{12}$ can be justified. In addition, as $\eta_{i, i+1}$ is the $\mathrm{LC}$ efficiency provides much more physical insight than $\varphi_{i}$.

It should be mentioned that some other $J_{02}$ temperature dependences such as $J_{02} \propto T^{5 / 2} \exp \left(\frac{-E_{z}}{2 k T}\right)$ may be found in the literature $[2,23-25]$. Because the $J_{02}$ parameter is related to the space charge recombination through defects, its temperature dependence is determined by the nature of those defects in each junction as well as by the bias conditions considered in its derivation. Therefore, it is reasonable to find different temperature dependences for $J_{02}$. If we consider the proportionality of $J_{02}$ with $T^{5 / 2}$ then $\varphi_{1}^{2}(T) \propto T^{2}$. Because $\varphi_{1}$ is a very sensitive parameter (no exponential dependence), this temperature dependence also leads to $\varphi_{1}$ values that lie between the ranges of values (colored regions of Figure 6.) that achieve good data fittings to the experimental measurements of Figures 4 and 5 .

\section{ANALYSIS OF THE SUBCELLS QUALITY}

\subsection{Subcell analysis from dark IV models and internal voltages}

A simplified two diode model has been used to analyze the behavior of each subcell as a function of temperature. This model is also useful to confirm the saturation currents employed for the calculation of $\varphi_{i}(T)$. Because the cells have a very high quality (as shown by the presence of strong LC) and the EL measurements are not affected by series resistance in the measurement range, neither shunt nor series resistance was considered in the model. The recombination current at each subcell as a function of voltage and temperature can be expressed as

$$
J(V, T)=J_{01}(T) e^{\frac{q V}{k T}}+J_{02}(T) e^{\frac{q V}{2 k T}}
$$

where $J_{01}$ represents the saturation current densities at the quasi-neutral regions under low-level injection conditions and includes radiative and non-radiative processes, $J_{02}$ represents the saturation current density associated to the depletion and perimeter regions, the other variables have their usual meaning. The main dependence of the recombination current in the neutral regions on the applied voltage $(V)$ is given by the dependence with the voltage of the injection of minority carriers from the depletion region into the neutral region (exponential dependence). Therefore, the ideality factor $(n)$ in the first term of expression (6) is always one regardless of the predominant recombination mechanism in the neutral regions, radiative, ShockleyRead-Hall (SRH) or Auger. The recombination current in the depletion region is obtained by integrating the different recombination mechanisms (SRH, radiative, and Auger) rates along it. Usually, only the SRH recombination mechanism is considered in this region in the literature [26] because at forward bias capture processes are the major recombination-generation phenomena in the depletion region [22]. When the SRH recombination in 
the depletion region takes place through a single-level trap, assuming that the trap is located in the center of the band gap and the capture cross section of electrons and holes are equal, the maximum value of the recombination occurs where the electron and hole densities are equal. In this case, the ideality factor $n$ is two. Although we have observed that in some cases this ideality factor can be lower than two, in this work we assume it is two in accordance with the models for LC available at this moment. Extended models which include the possibility of using different ideality factors for the depletion region recombination current are under development, and they will be used in future works [14].

The dark J-V curves of the individual junctions of the tandem cells can be extracted from EL data [16] to experimentally determine $J_{01}$ and $J_{02}$. Errors in these EL results are reduced when the $\mathrm{LC}$ is also considered $[14,18]$. An excellent agreement between the subcell dark $\mathrm{J}-\mathrm{V}$ curves extracted from EL and the model has been achieved at each temperature. The saturation currents have been validated by comparing the saturation currents used to calculate $\varphi_{1}(T)$ from Eq. (6) and the saturation currents extracted from the fits to the experimental dark J-V curves of each subcell as can be seen in Figures 7 and 8 .

Therefore, a proportionality factor for $\varphi_{1}^{2}(T)$ can be extracted from Eq. (6) by using the saturation current values of Figures 7 and 8 , giving $1.85 \cdot 10^{-4} \mathrm{~mA} \cdot \mathrm{cm}^{-2} / \mathrm{K}$ for GaInP in the ML732 device and $6.34 \cdot 10^{-3}$ $\mathrm{mA} \cdot \mathrm{cm}^{-2} / \mathrm{K}$ for GaAs in the MM708 device. The proportionality factor does not show a value that can be applicable to all materials. In fact, it is considerably higher for GaAs in the MM708 device indicating a high contribution from the $J_{2}$ term.

Once the saturation currents values have been validated, they can be used to evaluate the quality of the subcells in each structure. We analyze two different curves as a function of temperature: (1) the bandgap-voltage offset under open circuit conditions, $W_{o c}=\frac{E_{g}}{q}-V_{o c}$ [27]; and (2) the $V_{\mathrm{oc}}-V_{\mathrm{p}}$, where $V_{\mathrm{p}}$ corresponds to the parity voltage

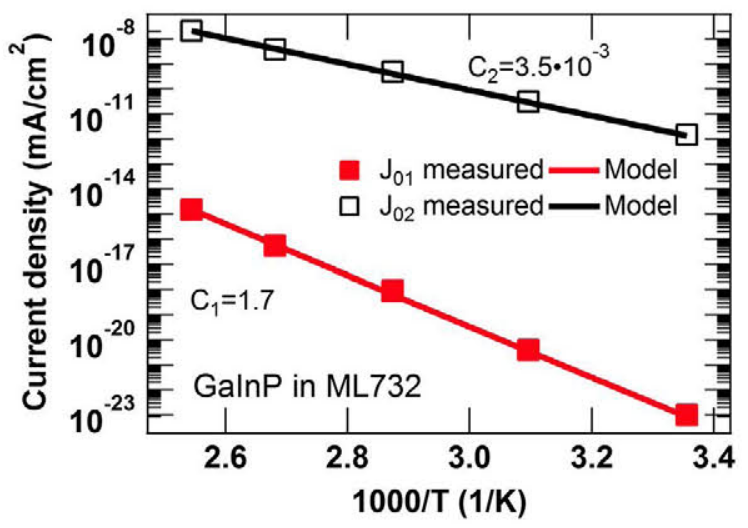

Figure 7. $J_{01}$ and $J_{02}$ for GalnP in the ML732 device. $C_{1}$ and $C_{2}$ (in units of $\mathrm{mA} / \mathrm{cm}^{2}$ ) correspond to the proportionality constants of Eqs. (3) and (4), respectively.

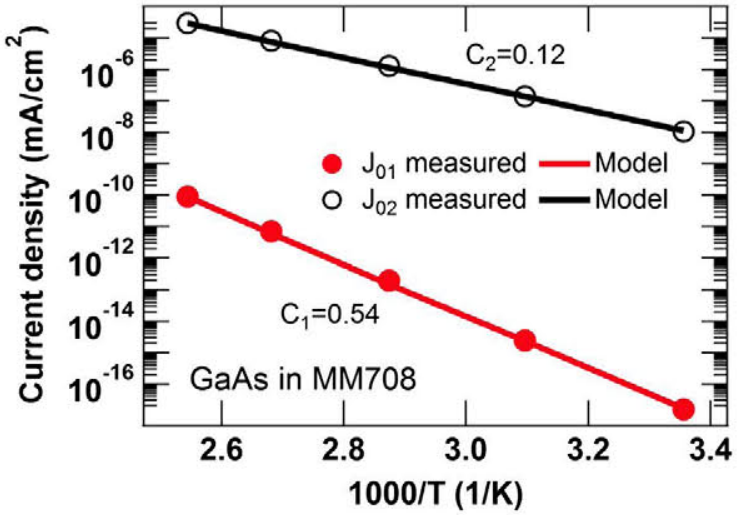

Figure 8. $J_{01}$ and $J_{02}$ for GaAs in the MM708 device. $C_{1}$ and $C_{2}$ (in units of $\mathrm{mA} / \mathrm{cm}^{2}$ ) correspond to the proportionality constants of Eqs. (3) and (4), respectively.

point where both the recombination current density in the quasi-neutral regions $\left(J_{1}\right.$, first term of Eq. (7)) and in the depletion and perimeter regions $\left(J_{2}\right.$, second term of Eq. (7)) are equal [28]. To base the model on realistic data, the $V_{\text {oc }}$ and bandgap values of each material are extracted from the EL measurements following the procedure of [18]. In Figure 9, we plot the difference between the $V_{\text {oc }}$ of the subcell at one sun and $V_{p}$ and the bandgap-voltage offset.

A positive $V_{\mathrm{oc}}-V_{\mathrm{p}}$ positive means that the recombination current density in the quasi-neutral regions starts to dominate before it reaches the $V_{o c}$ of the subcell. A negative value means that the recombination at the depletion and perimeter regions is still strongly affecting the performance of the device at the $V_{o c}$ point. This second case does not apply to any subcell analyzed, as can be seen in Figure 9. The higher the positive difference the lower the impact of the recombination at the space charge region and, thus, the better the quality of the subcell. The bandgap-voltage offset is similar in both cases. The $W_{o c}$ decreases at a rate of $-1.28 \mathrm{mV} / \mathrm{K}$ and $-1.14 \mathrm{mV} / \mathrm{K}$ for the GaInP top cell in the ML732 and the GaAs in the MM708, respectively. This indicates that GaInP is most

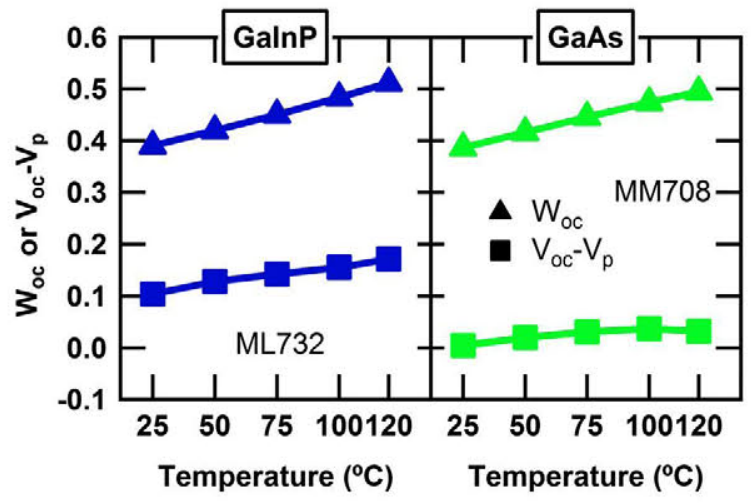

Figure 9. Temperature dependence of $W_{o c}$ and $V_{o c}-V_{p}$ for each top cell in the two structures analyzed. 
affected by temperature, but because $V_{\mathrm{oc}}-V_{\mathrm{p}}$ has a positive slope, it has lower influence of space charge recombination. This is consistent with having dominant $J_{01}$ (as in the case of the GaInP in the ML732) over the $J_{02}$ contribution to the total recombination current yield to higher temperature dependence $\left(J_{01} \propto T^{3}\right)$ as shown in Eqs. (4) and (5). The lower space charge recombination of the GaInP in the ML732 device can be attributed to the rear-heterojunction design [18]. In the case of the GaAs in the MM708 device, the $V_{\mathrm{oc}}-V_{\mathrm{p}}$ is also positive but closer to zero, and it remains almost constant with temperature indicating that at any temperature the space charge region recombination still limits the performance of the device.

\subsection{Subcell internal radiative efficiency dependence on the injected current and temperature}

The LC parameters are complementary, and incorrect conclusions can be drawn if the LC is evaluated by looking at them individually. In fact, in Section III, we have shown higher coupling efficiency factors for GaAs (MM708), but having higher coupling efficiency factor does not necessarily mean better luminescent properties ( $\eta_{12}$ depends also on the optical properties of the semiconductor structure). Therefore, the behavior of the internal radiative efficiency with injected current and temperature is used to provide more insight and to evaluate the luminescent properties of the junction materials. The internal radiative efficiency is defined as the fraction of recombination events that are radiative and is one of the most important metrics to determine the quality of the solar cells [20]. In order to calculate it, we use the optical modeling described in [12]. This modeling is capable of computing average internal probabilities of an emitted photon to be reabsorbed in the junction $\left(\overline{P_{a b s}}\right)$ and to escape out of the front surface of the device $\left(\overline{P_{\text {esc }}}\right) \cdot \eta_{\text {int }}$ can be defined from the optical modeling as

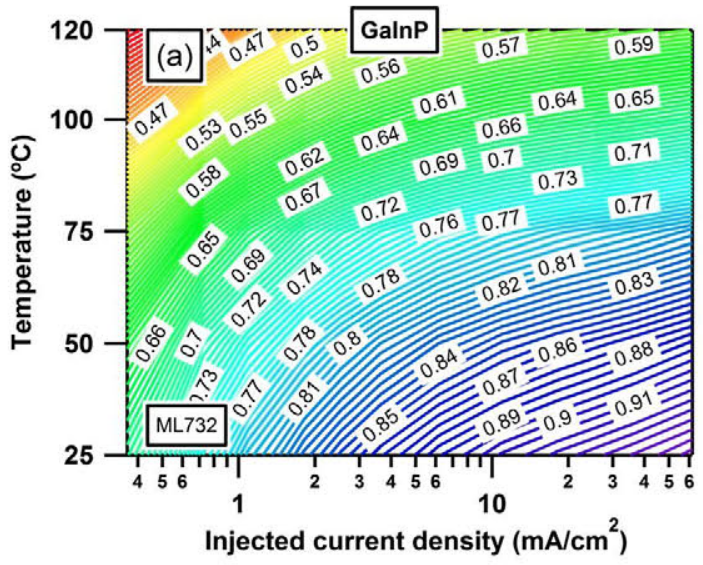

$$
\eta_{\text {int }}=\frac{\eta_{\text {ext }}}{\eta_{\text {ext }} \overline{P_{a b s}}+\overline{P_{e s c}}}
$$

where $\eta_{\text {ext }}$ is the external radiative efficiency, which is determined from EL measurements. The external radiative efficiency is defined as $\eta_{e x t}=\frac{J_{i}^{e m}}{J_{i}^{i n}}$, where $J_{i}^{e m}$ is the total external radiative flux from the $i^{\text {th }}$ junction in units of current density and is calculated by integrating over the EL peak emission spectrum and $J_{i}^{i n j}$ is the injected current density applied to the cell in the EL measurement $[14,18]$. $\overline{P_{e s c}}$ and $\overline{P_{a b s}}$ are complicated functions of the geometry and the angle and wavelength dependent Fresnel coefficients at the front and back of the structure. Their expressions are given in Ref. 12. To accurately determine $\eta_{\text {int }}$, reliable refractive indexes are needed. We have no measured data available for the extinction coefficient $(k)$ of the materials involved for temperatures above $25^{\circ} \mathrm{C}$. It was calculated by using the approach from [29] where the $k$ drops with the square root of the bandgap energy. The validation of this optical data has been performed by fitting the measured reflectance and $\mathrm{QE}$ response (not shown), reaching a satisfactory result. The determination of $\overline{P_{\text {esc }}}\left(1.26 \%\right.$ and $0.6 \%$ at $25^{\circ} \mathrm{C}$ for ML732 and MM708, respectively) and $\overline{P_{a b s}}\left(73 \%\right.$ and $84.2 \%$ at $25^{\circ} \mathrm{C}$ for ML732 and MM708, respectively) as a function of temperature does not show any significant changes, within $0.1 \%$ and $34 \%$, respectively from $25^{\circ} \mathrm{C}$ to $120^{\circ} \mathrm{C}$ for each top cell examined. It is reasonable to expect $\overline{P_{a b s}}$ and $\overline{P_{e s c}}$ to remain almost unchanged in the temperature range analyzed because the QE and reflectance does not show any significant changes except at the bandgap regions (Figures 2 and 3). Therefore, the variation in $\eta_{i n t}$ is dominated by the variation in $\eta_{\text {ext }}$. Figure 10 shows contour plots of the $\eta_{\text {int }}(J, T)$ for the subcells emitting light in each device (GaInP for the ML732 and GaAs for the MM708 device), as calculated from Eq. (8) and using EL data measured earlier. As can be seen in Figure 10a, we find a dominant radiative component for the $\mathrm{GaInP}$ (high values of $\eta_{\text {int }}$ even at low currents). $\eta_{\text {int }}(J, T)$ increases with the

Figure 10. Contour plot of $\eta_{\text {int }}(J, T)$ of the top cells: (a) GalnP in ML732 (b) and GaAs in MM708.

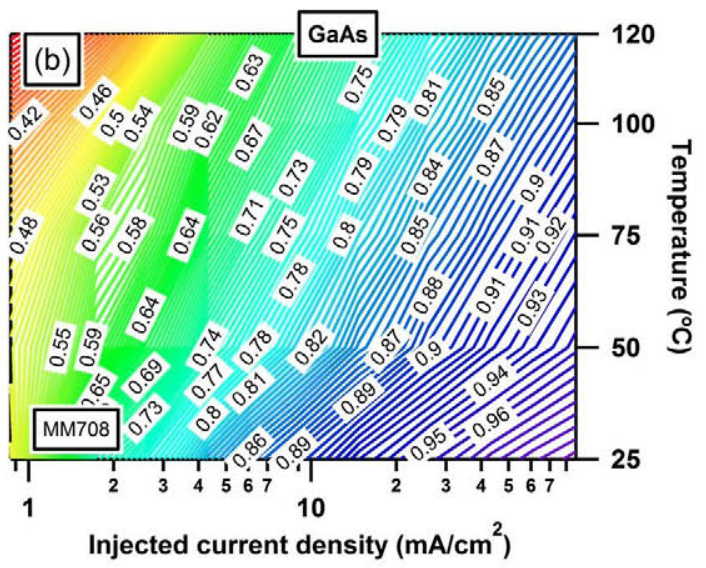


injected current, but it exhibits a strong reduction with temperature.

For the GaAs top cell in Figure $10 \mathrm{~b}, \eta_{\text {int }}(J, T)$ also decreases with temperature but at a lower rate than the GaInP top cell for any range of injected current. The higher sensitivity to the injected current suggests that at lower currents, the GaAs is still affected by a strong nonradiative recombination, which decreases at higher currents. It is noteworthy that at high current densities $\left(\sim 86 \mathrm{~mA} / \mathrm{cm}^{2}\right.$ or $\sim 7$ suns) and at any temperature below $100^{\circ} \mathrm{C}$, the GaAs subcell has very high values of $\eta_{i n t}(J, T)$ that exceed $90 \%$.

The internal emission intensity shown in Figure 10 can be affected by several mechanisms such as non-radiative recombination via deep level defects, surface recombination, potential losses in heterostructure barriers, optical losses at the interfaces, cladding layers and tunnel junctions, and so on. The temperature dependence of these processes is not a straightforward calculation. However, all those mechanisms can be included in the radiative and non-radiative lifetimes: $\tau_{r}$ and $\tau_{n r}$. Accordingly, the internal radiative efficiency can be also defined in terms of the radiative and non-radiative lifetimes [30]:

$$
\eta_{\text {int }}=\frac{\tau_{r}^{-1}}{\tau_{r}^{-1}+\tau_{n r}^{-1}}
$$

The temperature dependences of $\tau_{r}$ and $\tau_{n r}$ are wellknown and can be introduced into Eq. (9). From the bimolecular expression of radiative transitions, the $B$ radiative coefficient has weak temperature dependence: $\propto T^{-3 / 2}$ [30]. The dependence of the radiative lifetime is inversely proportional to $B$ for a specific doping level. In the case of the non-radiative recombination lifetime (the Auger recombination is neglected), the temperature dependence is stronger: $\tau_{n r}^{-1} \propto e^{\frac{-E a}{k T}}$ [30]. By combining the temperature dependences of the lifetimes into Eq. (9), a simple equation for the temperature dependence of the $\eta_{\text {int }}$ can be expressed as

$$
\eta_{\text {int }}(T)=\frac{\tau_{r, T_{0}}^{-1}\left(\frac{\mathrm{T}}{T_{0}}\right)^{-\frac{3}{2}}}{\tau_{r, T_{0}}^{-1}\left(\frac{\mathrm{T}}{T_{0}}\right)^{-\frac{3}{2}}+\tau_{n r, T_{0}}^{-1} \exp \left(\frac{-E_{a}}{k T}+\frac{E_{a}}{k T_{0}}\right)}
$$

where $T_{0}$ corresponds to a given reference temperature, in this case we use $300 \mathrm{~K}$, and $E_{a}$ is the activation energy of the trap. The exponential dependence of the nonradiative recombination is much stronger than the power law dependence, so the reduction of $\eta_{\text {int }}$ is due to a decrease in the non-radiative lifetime. In Figure 11, we show the values of $\eta_{\text {int }}$ at one sun-equivalent injected current as obtained from the optical modeling and the fitting to Eq. (10).

The trend can be explained by using Eq. (10) in all materials. As mentioned before, because the exponential behavior of the non-radiative lifetime has stronger temperature dependence than the radiative lifetime which follows a power law; the changes on the non-radiative

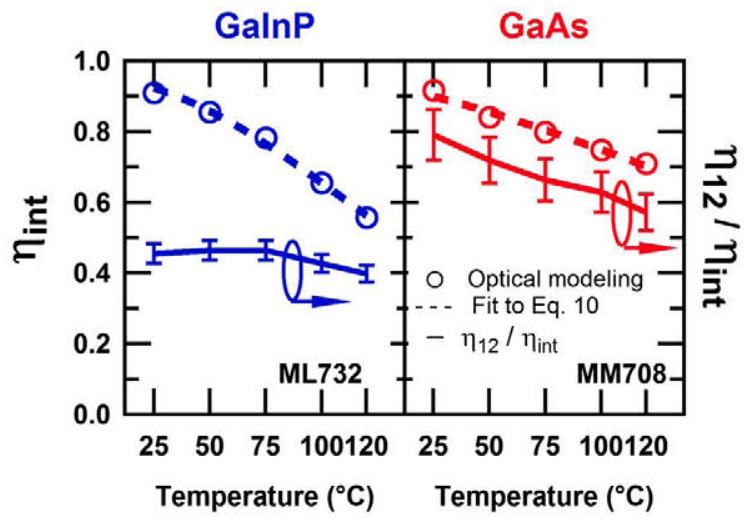

Figure 11. Internal radiative efficiencies at one sun-equivalent injected current calculated by optical modeling described in [12]. Dashed lines are the corresponding fitting curves calculated with Eq. (10).

Table I. IFitting parameters of Eq. (10).

\begin{tabular}{llcr}
\hline Junction & Structure & $\boldsymbol{\tau}_{r, T_{0}} / \boldsymbol{\tau}_{n r, T_{0}}$ & $E_{a}(\mathrm{meV})$ \\
\hline GalnP & ML732 & 0.086 & $196 \pm 14$ \\
GaAs & MM708 & 0.115 & $97 \pm 14$ \\
\hline
\end{tabular}

lifetime values dominate the reduction of $\eta_{\text {int }}$ as a function of the temperature. In fact, the $\tau_{n r}$ is more than one order of magnitude lower at $120^{\circ} \mathrm{C}$ than at $25^{\circ} \mathrm{C}$, while the $\tau_{r}$ increases slightly with temperature. The activation energy is the most important parameter in predicting the trend of the curve. The lower the activation energy is the lower the temperature dependence on the reduction of the internal radiative efficiency. Interestingly, the ratio $\tau_{r, T_{0}} / \tau_{n r, T_{0}}$ shown in Table I indicates that the effective carrier lifetime is dominated by the radiative lifetime at the reference temperature in both materials, being more dominant for the GaInP.

\section{CONCLUSIONS}

The temperature dependence of the LC of multi-junction solar cells has been studied in two tandem solar cells (GaInP/GaAs and GaAs/GaInAs) that are components of an inverted metamorphic 3J solar cell. The study has been carried out by means of LC analysis, dark modeling and optical modeling. LC models have shown suitability for the analysis of the impact of temperature on the luminescent coupling. This analysis showed that the LC efficiency factor is reduced with temperature from test temperature to typical operating point $\left(75^{\circ} \mathrm{C}\right)$ by $11 \%$ and $25 \%$ for GaInP and GaAs, respectively. From optical modeling, the luminescent properties of $\mathrm{GaInP}$ and $\mathrm{GaAs}$ subcells as a function of temperature and injected currents have also been studied. In the case of GaInP, it shows a high internal radiative efficiency even at low current densities, but it is 
strongly affected by the temperature that can be attributed to the dominant $J_{01}$ in accordance with dark modeling. The internal radiative efficiency of the GaAs top cell performs better with temperature and reaches very high values at moderate injected currents. The low values of internal radiative efficiency of this GaAs top cell at low current densities suggest a strong non-radiative recombination component. In both materials, there is strong potential to mitigate the effect of temperature by exploiting the LC properties at high current densities or light concentration levels.

\section{ACKNOWLEDGEMENTS}

M. Ochoa is grateful with all support from people at NREL during his research stay and for the scholarship granted by the Technical University of Madrid (UPM) through the program: Ayudas para estancias breves en España y en el extranjero. I. García holds an IOF grant from the People Programme (Marie Curie Actions) of the European Union's Seventh Framework Programme (FP7/ 2007-2013) under REA Grant Agreement no. 299878. This work has been supported by the US Department of Energy under Contract no. DE-AC36-08GO28308 with the National Renewable Energy Laboratory, by the Spanish MINECO project TEC2014-54260-C3-1 and by the Comunidad de Madrid (MADRID-PV P2013/MAE-2780).

\section{REFERENCES}

1. Espinet-González P, Algora C, Núñez N, Orlando V, Vázquez M, Bautista J, Araki K. Temperature accelerated life test on commercial concentrator III-V triplejunction solar cells and reliability analysis as a function of the operating temperature. Progress in Photovoltaics: Research and Applications 2015; 23(5): 559-569.

2. Siefer G, Bett AW. Analysis of temperature coefficients for III-V multi-junction concentrator cells. Progress in Photovoltaics: Research and Applications 2014; 22: 515-524.

3. Algora C. Very-high-concentration challenges of III-V multijunction solar cells. In Chapter 5 of the Book "Concentrator Photovoltaics", Vol. 130 Luque A, Andreev V (eds). Springer: Heidelberg, Germany, 2007; 89-111. ISBN:978-3-540-68796-2.

4. Rodrigo P, Fernández EF, Almonacid F, PérezHigueras PJ. Review of methods for the calculation of cell temperature in high concentration photovoltaic modules for electrical characterization. Renewable and Sustainable Energy Reviews 2014; 38: 478 488.

5. Kinsey GS, Hebert P, Barbour KE, Krut DD, Cotal HL, Sherif RA. Concentrator multijunction solar cell characteristics under variable intensity and temperature. Progress in Photovoltaics: Research and Applications 2008; 16: 503-508.
6. NREL Press release: "NREL demonstrates $45.7 \%$ efficiency for concentrator solar cells", http://www. nrel.gov/news/press/2014/15436.html [accessed on 16 December 2014].

7. MA Steiner, Geisz JF, Friedman DJ, Olavarria WJ, Duda A, Moriarty TE. Temperature-dependent measurements of an inverted metamorphic multijunction (IMM) solar cell. In Photovoltaic Specialists Conference (PVSC), 2011 37th IEEE, June 2011; 2527-2532.

8. Friedman DJ, Geisz JF, Steiner MA. Analysis of multijunction solar cell current-voltage characteristics in the presence of luminescent coupling. Photovoltaics, IEEE Journal of 2013; 3(4): 1429-1436.

9. Friedman DJ, Geisz JF, Steiner MA. Effect of luminescent coupling on the optimal design of multijunction solar cells. Photovoltaics, IEEE Journal of 2014; 4(3): $986-990$.

10. Steiner MA, Geisz JF. Non-linear luminescent coupling in series-connected multijunction solar cells. Applied Physics Letters 2012; 100: 251106.

11. Steiner MA, Geisz JF, Moriarty TE, France RM, McMahon WE, Olson JM, Kurtz SR, Friedman DJ. Measuring IV curves and subcell photocurrents in the presence of luminescent coupling. Photovoltaics, IEEE Journal of 2013; 3: 879-887.

12. Steiner MA, Geisz JF, García I, Friedman DJ, Duda A, Kurtz SR. Optical enhancement of the open-circuit voltage in high quality GaAs solar cells. Journal of Applied Physics 2013; 113(12): 123109.

13. Geisz JF, Steiner MA, García I, Kurtz SR, Friedman DJ. Enhanced external radiative efficiency for $20.8 \%$ efficient single-junction GaInP solar cells. Applied Physics Letters 2013; 103: 041118.

14. Geisz JF, Steiner MA, García I, France RM, McMahon WE, Osterwald CR, Friedman DJ. Generalized optoelectronic model of series-connected multijunction solar cells. IEEE Journal oh Photovoltaics 2015; 5(6): 1-13.

15. Geisz JF, Kurtz S, Wanlass MW, Ward JS, Duda A, Friedman DJ, Olson JM, McMahon WE, Moriarty TE, Kiehl JT. High-efficiency GaInP GaAs InGaAs triplejunction solar cells grown inverted with a metamorphic bottom junction. Applied Physics Letters 2007; 91(2): 90-93. 023502.

16. Kirchartz T, Rau U, Hermle M, Bett AW, Helbig A, Werner JH. Internal voltages in GaInP GaInAs Ge multijunction solar cells determined by electroluminescence measurements. Applied Physics Letters 2008; 92(12): 90-93.

17. Rau U. Reciprocity relation between photovoltaic quantum efficiency and electroluminescent emission of solar cells. Physical Review B 2007; 76: 085303.

18. Geisz JF, France RM, García I, Steiner MA, Friedman DJ. Device characterization for design optimization of 
4 junction inverted metamorphic concentrator solar cells. In AIP Proceedings of the 10th International Conference on Concentrator Photovoltaic Systems, Albuquerque, NM; 2014.

19. Osterwald CR, Emery KA, Myers DR, Hart RE. Primary reference cell calibrations, at SERI: history and methods. In Photovoltaic Specialists Conference. Conference Record of the Twenty First IEEE, May 1990; 2: 1062-1067.

20. Green MA. Radiative efficiency of state-of-the-art photovoltaic cells. Progress in Photovoltaics: Research and Applications 2012; 20(4): 472-476.

21. Nell ME, Barnett AM. The spectral pn junction model for tandem solar-cell design. Electron Devices, IEEE Transactions on $1987 ; 34$ (2): 257-266.

22. Sze SM, Ng KK. Physics of Semiconductor Devices. John Wiley \& Sons, Inc.: Hoboken, NJ, USA, 2006.

23. Wolf M, Noel GT, Stirn RJ. Investigation of the double exponential in the current-voltage characteristics of silicon solar cells. Electron Devices, IEEE Transactions on 1977; 24(4): 419-428.

24. Reinhardt KC, Yeo YK, Hengehold RL. Junction characteristics of $\mathrm{Ga0} .5 \operatorname{In} 0.5 \mathrm{P} \mathrm{n}+\mathrm{p}$ diodes and solar cells. Journal of Applied Physics 1995; 77(11): 5763-5772.
25. Broderick LZ, Albert BR, Pearson BS, Kimerling LC, Michel J. Design for energy: modeling of spectrum, temperature and device structure dependences of solar cell energy production. Solar Energy Materials and Solar Cells 2015; 136: 48-63.

26. Luque A, Hegedus S. Handbook of Photovoltaic Science and Engineering. John Wiley \& Sons: Chichester, UK, 2011.

27. King RR, Bhusari D, Boca A, Larrabee D, Liu X-Q, Hong W, Fetzer CM, Law DC, Karam NH. Band gap-voltage offset and energy production in nextgeneration multijunction solar cells. Progress in Photovoltaics: Research and Applications 2011; 19(7): 797-812 [Online].

28. Hoheisel R, Dimroth F, Bett AW, Messenger SR, Jenkins PP, Walters RJ. Electroluminescence analysis of irradiated $\mathrm{GaInP} / \mathrm{GaInAs} / \mathrm{Ge}$ space solar cells. Solar Energy Materials and Solar Cells 2013; 108: 235-240, Selected publications from the 22nd Space Photovoltaic Research and Technology (SPRAT) Conference.

29. Pankove JI, Optical processes in semiconductors: Dover, 1971.

30. Schubert EF. Light-Emitting Diodes, 2nd ed. Cambridge University Press: Cambridge, UK, 2006. 